\title{
Challenges of Advanced Analytics Maturity Model Development
}

\author{
Santa Lemsa \\ Department of Economics and Business \\ Vidzeme University of Applied Sciences \\ Riga, Latvia \\ santa.lemsa@va.lv
}

\begin{abstract}
Significance to understand the advanced analytics ecosystem maturity is increasing caused by constantly growing data volumes and demand for advanced analytics including automated decision making based on data or process automation. The analytics maturity assessment helps to identify strengths and weaknesses of the organization's analytics ecosystem and can provide detailed action plan to move to the next level. The focus of the paper is to review and analyse analytics maturity models to assess their application as frame to build a new analytics maturity model or replicate with time adjustment any of reviewed models. The literature review and publicly available assessment models provided by analytics sector were used to review and analyse analytics maturity models. Fifteen models were reviewed and four of them analysed by twelve characteristics. Summary of four models includes analytics maturity levels, domains, accessibility of questionnaire, discloser of maturity level detection and authors assessment of several characteristics. Comprehensive descriptions of analytics maturity levels were available for many models. Solid recommendation sets for each maturity level provided for the most disclosed models. One of the most important components, approach to detect specific maturity level, was not transparent or disclosed with limitations. However, it is possible to develop a new model or replicate in some extent based on models reviewed in this paper, but it requires extensive professional experience in advanced analytics and related functions.
\end{abstract}

Keywords - advanced analytics, analytics maturity, maturity models, maturity assessment.

\section{INTRODUCTION}

Every minute of every day a huge amount of data created - social media, email communication, any device connected to the internet, google search. The future of digitization and internet of things promise a further generation of new data volumes. New advanced analytical approaches demanded to deal with and make sense out of large volumes of unstructured and structured data.
Data-driven business environment is competitive advantage for any organization. To ensure faster and smarter decision-making, organizations are forced to use advanced analytics to analyse the past, understand the present behaviour and predict and influence the future events, actions, decisions and behaviour. The potential value of data is uncovered only when data-driven decision making becomes a culture of organizations like a blood circulation. Several studies argue that in order to establish data driven decision making, - organizations need to introduce maximally automated processes to manage and use all different kind and fast-moving data from internal and external sources to turn that information into deep and colourful insights [1]. New approaches, algorithms, tools and platforms help to make sense out of large volumes of unstructured and structured data, and methods which ensure so called advanced analytics [2].

The competition between organizations is very tough and usually requests many decisions on organization's side before launching the product or communication with potential customers. The one of differentiators of success is an ability to make decisions which support customers' values and preferences. To ensure faster and smarter decision-making, organizations are forced to use advanced analytics to analyse the past, understand the present behaviour and predict and influence the future events, actions, decisions and behaviour. By implementing advanced analytics into operations, organizations significantly increase a control over daily decisions that ensures a higher potential to meet their business goals [3].

Assessment of the advanced analytics ecosystem is crucial for further development, competitions in the market and to reach the strategic goals of the organization. The assessment and understanding of the investments needed and next steps is critical to make digitization process productive [4]. 
Considering the increasing demand for advanced analytics including automated decision making based on data or process automation the significance to understand the advanced analytics ecosystem maturity level in any country, industry or organization is topical. The analytics maturity assessment or detection of the analytics development level by several factors which are crucial to ensure proper analytics performance helps to identify strengths and weaknesses of the organization's analytics ecosystem and can provide detailed action plan step by step to move existing analytics ecosystem to the next level or level what is relevant to the organization to meet its strategic goals. However, we can find the models for advanced analytics maturity assessment, there is a limited disclosure of the specific methodology how to develop such models. The assessment process, specific factors and their weight to put organization in the specific level of analytics maturity, are more 'know-how' of analytics sector than transparently disclosed full methodology to ensure reproducibility or validation of the models. Another issue is a time, data volumes and rapid development of technologies what requires regular adjustment of the model.

The paper aims to enhance previous reviews of the models with insights of the methodology and overall process to build or replicate such assessment models. Various organizations' analytics maturity models are publicly available with, in some level, disclosed methodology to find out domains or spheres of influence, factors, questions, answers, behaviour and drivers what allows to build such assessment model. 15 models reviewed and 4 analysed in this paper.

\section{MATERIALS AND METHODS}

The review and analysis of the analytics maturity models is based on the literature review - scientific publications, reports of the researches, books published by experts and opinion leaders, online published materials and practical assessment of the publicly available analytics maturity assessment tools provided by analytics, technical or IT consulting companies. The literature review process was performed in 2 stages - identified, collected and reviewed materials to point out analytics maturity models with the most extensive information about methodology behind the development of models, the most trustable, the most known and widely used. The second stage was practical experiment taking the online tests to assess the organizations' analytics maturity level to complement existing description of the models from the first stage of literature review. As a result, summary of the characteristics was created for the 4 models who can serve as a base to replicate, adjust or build the own model for specific region, country, industry or segment.

\section{RESULTS AND DISCUSSION}

\section{A. Analytics maturity models}

Many maturity models for Analytics, Business Analytics, Business Intelligence, Big data, Information Systems have been developed with several domains and stages of analytics. One of the first is Watson's model for data warehousing maturity which covers such domains like people, processes and technologies [5]. Comuzzi \& Patel provides an explicit summary of domains and subdomains used in different maturity assessment models. There are such domains as Analytics, Organization, Governance, Technologies, Data, Sponsorship, Culture, Strategy, People and others that have been used and proposed by several authors [6]. Davenport \& Harris's Analytics maturity assessment model proposed in 2007 is one of the very often cited ones and used as a base. They indicate 3 domains: 1 ) Organization with subdomains Analytical objectives, Analytical processes; 2) Human with subdomains Skills, Sponsorship, Culture, and 3) Technology [4]. In 2010, the base model was enhanced with DELTA framework [8] and in 2017 the new release followed with DELTA Plus model containing 7 domains: Data, Enterprise, Leadership, Targets, Analysts, Technology, Analytics techniques and 5 maturity stages from Analytically Impaired to Analytical Competitors [9]. Krol \& Zdonek disclose a rich summary of analytics maturity models with stages and domains [7]. Cosic et al. proposes 4 domains with 4 subdomains for each: “Governance (Decision Rights, Strategic Alignment, Dynamic BA Capabilities, Change Management), Culture (Evidence-based Management, Embeddedness, Executive Leadership and Support, Flexibility and Agility), Technology (Data Management, Systems Integration, Reporting and Visualisation BA Technology, Discovery BA Technology), People (Technology Skills and Knowledge, Business Skills and Knowledge, Management Skills and Knowledge, Entrepreneurship and Innovation)” [10].

Traditionally 5 levels of maturity are used widely, sometimes the Zero level is created to sort those who haven't done/ implemented/ developed anything in specific area. Becker at al. [11] identified and worked out 5 levels of maturity. All the above-mentioned authors use a 5-level maturity assessment. In case of Comuzzi \& Patel [6], in addition, the Zero level had been created because of organizations that required sorting them as the Zero level in a specific domain or subdomain.

Summarizing the authors above, the 5 levels of maturity can be interpreted: level 1 - beginners with weak analytical capability, only spreadsheet based and issues with data gathering and quality; level 2 - intermediate silos analytical activities, better data accessibility; level 3 - wide operational usage with some coordination between analytical community, existing data warehouses/ repositories/data lakes; level 4 - analytical organization with high quality data, integrated analytics in the many processes and decision-making, analytics as competitive advantage; level 5 - visionary advanced organizations with analytics culture and mindset, testing/adopting cutting edge tools/techniques/solutions, high competitive advantage.

Exploring analytics maturity models to gather and analyse the methodology behind to build or replicate such model, the 15 analytics maturity models were reviewed: 1 . Watson's data warehousing maturity [5], 2. Comuzzi \& Patel model [6], 3. Early DELTA model [4], 4. Cosic et al BACMM model [10], 5. Analytic Processes Maturity Model (APMM) [12], 6. Analytics Maturity Quotient Framework (AMQ) [13], 7. Blast Analytics Maturity Assessment Framework [14], 8. Data Analytics Maturity 
Environment. Technology. Resources. Rezekne, Latvia Proceedings of the $13^{\text {th }}$ International Scientific and Practical Conference. Volume 2, 88-92

Model for Associations (DAMM) [15], 9. DELTA Plus Model [16], 10. Logi Analytics Maturity Model [17], 11. Online Analytics Maturity Model (OAMM) [18], 12. SAS Analytics Maturity Scorecard [19], 13. TDWI Analytics Maturity Model [20], 14. Web Analytics Maturity Model WAMM [21], 15. Defining analytics maturity indicators (DAMI) [22].

After the review of literature, reports, publications and the test of publicly available assessment tools, 4 models were selected for deeper analysis. They were selected by such factors like discloser of the survey questions, described methodology, provided guidelines to replicate or adopt such model.

\section{B. Methodology behind Analytics maturity models}

The detailed methodology is necessary to replicate or adjust the model for the specific industry, country, segment or organization. Therefore, 4 models who disclose more explicit approach chosen for detailed analysis. Models were described by domains, factors, interpretation of the results (maturity level) and recommendations, any other supportive information what increases the ability to replicate or build the model. The summary of characteristics for the 4 models provided in Table 1 . In addition, authors own ranking of the following characteristics: Maturity level description, Recommendations, Reproducibility, Interpretation were used to indicate how helpful model could be for replication or development of analytics maturity model. 5-point ranking was used, where 1 - slightly helpful, with minimal description and 5 - very helpful with very detailed description. These are Analytics Maturity Quotient Framework (AMQ) [13], DELTA Plus Model [16], Defining analytics maturity indicators (DAMI) [22] and TDWI Analytics maturity model [20]

\section{Analytics Maturity Quotient Framework (AMQ)}

Analytics Maturity Quotient (AMQ) Framework is based on 4 domains: Data Maturity, Leadership, Analytics Talent, Decision making process. Domains contain 2-4 factors with 10-point scale. Final result as a Score between 0-10. Publicly available simple DYI survey [13] to assess AMQ and comprehensive AMQ assessment available for organizations. Model developed based on detailed stakeholder interviews, auditing and surveys. In case of comprehensive approach, the detailed and the prioritized recommendation set for increasing analytics maturity provided. The analytics maturity assessment model constantly is developed and updated.

\section{DELTA Plus Model}

DELTA Plus Model Adapted from [8],[4],[9] and is a tool developed by the International Institute for Analytics (IIA). DELTA Plus Model is based on 7 domains: Data breadth, integration, quality; Enterprise - approach to managing analytics; Leadership - passion and commitment; Targets - first deep then broad; Analysts professionals and amateurs; Technology - approach, orientation, velocity; Analytics techniques - sophistication, diversity. Publicly available version [24] provides 1 factor with 5 statements for each domain. Analytics maturity stage is provided between 1-5. 1 - Analytically Impaired, 2 - Localized Analytics, 3 - Analytical Aspirations, 4 Analytical Companies, 5 - Analytical Competitors. The comparison to the industry and digital native is provided complementary. The algorithm of the maturity level detection is not disclosed, but for those who make an assessment the explanation of each maturity stage is sent, the action list to move from one stage to another is shared. The model is developed on many years of researches, interviews, surveys.

\section{Defining analytics maturity indicators (DAMI)}

Defining analytics maturity indicators: A survey approach paper model is based on 5 domains: Data, Organization, Leadership, Techniques and applications and Analysts. 4 stages of the analytics maturity were found out with clustering based on 28 factors. 1 - No analytics, 2 analytics bootstrappers, 3 - sustainable analytics adopters, 4 - disruptive analytics innovators. The research provides key characteristics of each stage and key recommendations to improve analytics. Model developed based on interviews as a pre-test for the survey in 2 rounds with interval 1 year, results validation by experts. Full questionnaire is available with 67 questions.

\section{TDWI Analytics Maturity Model}

TDWI Analytics maturity model is based on 5 domains: Organization, Resource, Data Infrastructure, Analytics, Governance. Maturity consists of 5 stages plus 1 stage (chasm) between third and fourth stages. Maturity stages are 1 - Nascent, 2 - Early, 3 - Established, 4 - Mature, 5 Advanced/ Visionary and the Chasm - the most difficult stage to overcome to reach the next level. The research provides wide set of characteristics of each stage and solid outlook of recommendations to improve analytics. Model developed based on extensive researches, surveys and interviews for many years. Full questionnaire is available with 52 questions as online assessment tool. It is required to apply for the assessment to get full questionnaire [23].

All 4 models disclose domains and factors. Only AMQ model does not disclose specific maturity levels. 
TABLE 1 ANALYTICS MATURITY MODELS - SUMMARY OF CHARACTERISTICS

\begin{tabular}{|c|c|c|c|c|}
\hline Characteristics & AMQ & DELTA Plus & DAMI & TDWI \\
\hline Maturity levels & Not disclosed & 5 & 4 & $5+1$ \\
\hline Number of Domains & 4 & 7 & 5 & 5 \\
\hline Number of factors & 11 & 7 & 28 & 22 \\
\hline Assessment & 10-point scale & Statements & Scale, Statements & Scale, Statements \\
\hline Maturity level describer & AMQ score $0-10$ & DELTA Score 1-5 & Cluster 1-4 & Score $1-20$ \\
\hline Maturity level detection & Not disclosed & Not disclosed & Clustering & $\begin{array}{l}\text { Weighted score by } \\
\text { domains and average } \\
\text { total score }\end{array}$ \\
\hline Model development & $\begin{array}{l}\text { Interviews, auditing and } \\
\text { survey }\end{array}$ & $\begin{array}{l}\text { Researches, surveys, } \\
\text { interviews }\end{array}$ & $\begin{array}{l}\text { Interviews, survey, } \\
\text { validation by experts }\end{array}$ & $\begin{array}{l}\text { Researches, surveys, } \\
\text { interviews }\end{array}$ \\
\hline $\begin{array}{l}\text { Survey questionnaire } \\
\text { disclosed }\end{array}$ & $\begin{array}{l}\text { Short DIY version only, } \\
11 \text { questions }\end{array}$ & $\begin{array}{l}\text { Only } 7 \text { statements and } \\
\text { domains }\end{array}$ & 67 questions, full survey & 52 questions, full survey \\
\hline Maturity level description & 1 & 5 & 5 & 5 \\
\hline Recommendations & 1 & 5 & 3 & 1 \\
\hline Reproducibility & 3 & 4 & 5 & 5 \\
\hline Interpretation & 1 & 2 & 4 & 4 \\
\hline
\end{tabular}

Maturity level detection is not disclosed fully by any of models, however the algorithm behind is the most important to be able to build the model what is able to detect maturity level of specific organization. The most transparent is DAMI model. On the one hand, questionnaire is disclosed by all models, on another hand, publicly available versions for 2 models has very short questionnaires what could help to get some first estimation where organization stands overall, but would not be very helpful for new model development.

Author recognizes DAMI and TDWI models as the most appropriate to use as base for the new model development. These models could be the most helpful if person who aims to build own model does not have very extensive experience in wide range of analytics. These models provide full questionnaires and provides hints to make an analysis on the survey data which ensure some reproducibility of these models to use them in another countries or industries and afterwards allows to compare results.

\section{CONCLUSIONS}

Data, analytics, related tools and overall analytics ecosystem becomes more and more crucial topic in any organization taking into account high digitization demand. Therefore, organization's analytics maturity assessment becomes critical to continue successful business and many analytics service providers or analytics consulting companies develop analytics maturity assessment as part of their commercial services. There are much more analytics maturity assessment models available than mentioned in this paper, but very often publicly available versions are with limited options - fewer questions, not disclosed maturity level detection methodology, outcome as a highlevel assessment of maturity level what does not answers how, when, what, how much resources needed to make the next step in analytics maturity development.

Ability to build or replicate the own analytics maturity assessment model could be attractive to large organizations, organizations with existing analytical teams and drive to encourage analytical culture organizationwide, analytical teams, researchers, consultants and experts in analytics sector. Another reason is a rapid development of technologies, analytical platforms, increase of data volumes, data accessibility for wider audience what leads to risk - publicly available (not commercial) analytics maturity assessment models are outdated or partly outdated. However, models available in the market can provide comparison with industry, with similar segment, with overall level.

All 15 reviewed models give some skeleton for the independent development of the analytics maturity model. However, the person or group of persons who are going to build or replicate the model should be from the analytics or related industry - experts and/or practitioners, to be able not only replicate or build such model, but create relevant questionnaires, run audits, make interviews, understand, use and interpret outcome giving precise assessment of overall maturity level and by domains, develop a set of recommendations to improve existing level or move to the next. All reviewed models disclose domains, in some extent sub-domains or factors, at least high-level description of analytics maturity levels, but without discloser of methodology how to detect specific maturity level. In some cases, more information was provided what sits behind the model and how it was developed - like 
surveys, interviews with experts, audits, back test after some time on the same pool. From the 4 models analysed, 2 models disclose the full survey questionnaire, 1 give insight in analyses of the data and indication of the maturity levels, 2 gives some explanation on detection of maturity level.

It is possible to develop a new model or replicate in some extent analytics maturity assessment model based on models reviewed in this paper. The challenging part is methodology how to detect the level of maturity. Another challenge is to interpret results to provide explanation of detected analytics maturity level and recommendation for the next steps to improve overall analytics maturity level. One more challenge is to monetize the move to the higher maturity level. In addition, time and rapid development of technologies plays significant role because the model should include the newest trends of analytics ecosystem to not become outdated as soon it is created. It should be able to assess the maturity level properly today and in the midterm future to give the right recommendations to the organizations to develop the analytics ecosystem according to the newest and most applicable solutions. Thus, drives the need for the new and new analytics maturity assessment models.

\section{REFERENCES}

[1] A. Gandomi and M. Haider, "Beyond the hype: Big data concepts, methods, and analytics,” International Journal of Information Management, vol. 35, pp. 137-144, 2015.

[2] United States Government Accountability Office, "Data and Analytics Innovation. Emerging Opportunities and Challenges," September 2016. [Online]. Available: http://www.gao.gov/assets/680/679903.pdf. [Accessed: Mar. 21, 2021].

[3] C.V. Apte, S.J. Hong, R. Natarajan, E.P.D. Pednault, F.A. Tipu and S.M. Weiss, "Data-intensive analytics for predictive modeling," IBM Journal of Research \& Development, vol. 47 (1), pp. 17-23, 2003.

[4] T.H. Davenport and J.G.S. Harris, Competing on Analytics: The New Science of Winning. Harvard Business Press, 2007.

[5] H.J. Watson, "Recent Developments in Data Warehousing," Communications of AIS, vol. 8, pp. 1-25, 2002. [Online]. Available: https://aisel.aisnet.org/cais/vol8/iss1/1/. [Accessed March 18, 2021] https://doi.org/10.17705/1CAIS.00801

[6] M. Comuzzi and A. Patel, "How organisations leverage Big Data: a maturity model,” Industrial Management \& Data Systems, vol. 116(8), pp. 1468-1492, 2016.

[7] K. Krol and D. Zdonek, "Analytics Maturity Models: An Overview," Information vol. 11, p. 142, March 2020. [Online]. Available: https://www.mdpi.com/2078-2489/11/3/142. [Accessed October 18, 2020] https://doi.org/10.3390/info11030142

[8] T.H. Davenport, J.G. Harris, R. Morison, Analytics at Work: Smarter Decisions, Better Results. Harvard Business School Publishing, 2010.

[9] T.H. Davenport, J.G. Harris, Competing on Analytics: Updated, with a New Introduction: The New Science of Winning. Harvard Business Press, 2017.

[10] R. Cosic, G. Shanks and S. Maynard, Towards a Business Analytics Capability Maturity Model. In Proceedings of the 23rd Australasian Conference on Information Systems Business Analytics Capability, Dec 2012, Geelong.
[11] J. Becker, R. Knackstedt and J. Pöppelbuß, “Developing Maturity Models for IT Management - A Procedure Model and its Application,” Business \& Information Systems Engineering, vol. 1(3), pp. 213-222, 2009.

[12] R.L. Grossman, "A framework for evaluating the analytic maturity of an organization," International Journal of Information Management, vol. 38, pp. 45-51, 2018.

[13] J. Piyanka, “The Analytics Maturity Quotient Framework,” 2019. [Online]. Available: https://aryng.com/download/consultingdownloads/Aryng_-_Data_Culture_Assessment.pdf [Accessed: Mar. 22, 2021].

[14] Blast Analytics \& Marketing, “Analytics Maturity Assessment”, Blast Analytics \& Marketing, 2021. [Online]. Available: https://www.blastanalytics.com/analytics-maturity-assessment. [Accessed: Mar. 19, 2021].

[15] Association Analytics, " 5 Areas to Assess Using the DAMMData Analytics Maturity Model,” Association Analytics, 2017. [Online]. Available: https://associationanalytics.com/2017/12/29/5-areas-assess-dataanalytics-maturity-model/. [Accessed: Mar. 19, 2021].

[16] T.H. Davenport, "DELTA Plus Model \& Five Stages of Analytics Maturity: A Primer,” International Institute for Analytics, 2018. [E-book] Available: https://www.iianalytics.com/delta-plusprimer/. [Accessed Mar. 20, 2021].

[17] Logi Analytics, "The 5 Levels of Analytics Maturity: From Basic BI to Sophisticated Differentiators,” Logi Analytics, 2017. [Ebook] Available: https://go.logianalytics.com/ebook-maturitymodel-for-analytics-capabilities.html. [Accessed: Mar. 19, 2021].

[18] Cardinal Path, "How mature are your organization's digital analytics?,” Cardinal Path, 20. [E-book] Available: https://www.cardinalpath.com/collateral/oamm cp_wp.pdf. [Accessed: Mar.19, 2021].

[19] PharmaVOICE \& SAS, "Five Steps to Analytical Maturity. A Guide for Pharma Commercial Operations,” 2014. [Online]. Available:

https://www.sas.com/content/dam/SAS/en us/doc/whitepaper1/fiv e-steps-to-analytical-maturity-106929.pdf. [Accessed: Mar.19, 2021].

[20] F. Halper, “TDWI Analytics Maturity Model. Assessment Guide”, 2020. [Online]. Available: https://tdwi.ilumivu.com/org tdwi/media/other/TDWI Analytics Maturity_Model_Assessment_Guide_web.pdf. [Accessed: Mar. 22, 2021].

[21] S. Hamel, "The Web Analytics Maturity Model. A Strategic Approach Based on Business Maturity and Critical Success Factors,” 2009. [Online]. Available: http://www.cardinalpath.com/wpcontent/uploads/WAMM_ShortPaper_091017.pdf. [Accessed: Mar. 19, 2021].

[22] J. Lismonta, J. Vanthienen, B. Baesens and W. Lemahieua, "Defining analytics maturity indicators: A survey approach," International Journal of Information Management, vol. 37, pp. 114124, Jun. 2017. [Online]. Available: https://www.sciencedirect.com/science/article/pii/S026840121630 5655. [Accessed October 18, 2020], https://doi.org/10.1016/j.ijinfomgt.2016.12.003

[23] TDWI Assessment, "Analytics Maturity Model Assessment," 2020. [Online]. Available: https://tdwi.org/pages/assessments/advall-tdwi-analytics-maturity-model-assessment.aspx. [Accessed: Mar. 22, 2021].

[24] International Institute for Analytics, "Estimate Your Analytics Maturity With Our Free Tool”, 2018. [Online]. Available: https://www.iianalytics.com/ama-widget. [Accessed: Mar. 22, 2021]. 